\title{
Recent Advances in Molecular Imaging of Colorectal Tumors
}

\author{
Takanori Kashihara Naoki Muguruma Shota Fujimoto Yoshihiko Miyamoto \\ Yasushi Sato Tetsuji Takayama \\ Department of Gastroenterology and Oncology, Institute of Biomedical Sciences, Tokushima University Graduate \\ School, Tokushima, Japan
}

\author{
Keywords \\ Colorectal cancer · Molecular imaging · Fluorescence \\ endoscopy
}

\begin{abstract}
Background: Recent endoscopic studies have revealed that small colorectal tumors are often overlooked during colonoscopy, indicating that more sensitive detection methods are needed. Summary: Molecular imaging has received considerable attention as a new endoscopic technique with high sensitivity. It generally employs a fluorescence-labeled compound that specifically binds to a molecule on the tumor. Fluorescent probes for molecular imaging are largely classified as 2 types: a fluorescence-labeled antibody targeting a molecule specifically expressed on the tumor cell surface such as epidermal growth factor receptor or vascular endothelial growth factor (VEGF); and a fluorescence-labeled small molecule compound targeting a molecule specifically expressed in tumor cells including c-Met, glutathione S-transferase, $\mathrm{Y}$-glutamyltranspeptidase, cathepsin, or endothelin A receptor. These probes successfully detected colorectal tumors in several animal studies. Moreover, 3 recent human clinical trials evaluating endoscopic molecular
\end{abstract}

imaging for colorectal tumors have been reported. In one study, a Cy5-labeled synthetic peptide against c-Met was developed, and fluorescent endoscopic observation with this probe detected a greater number of colorectal adenomas than with white light observation. Another trial used IR800labeled anti-VEGF antibody, which sensitively detected human colorectal adenomas by fluorescent endoscopy. Last, a fluorescent probe with synthetic peptide against BRAF-positive cells was able to visualize sessile serrated lesions. The fluorescent probes accumulated at very high levels in colorectal tumor cells but at lower levels in surrounding nonneoplastic mucosa. Key Messages: We expect that molecular imaging techniques with fluorescent probes will soon lead to the establishment of a highly sensitive endoscopic method for colorectal tumor detection.

○ 2020 S. Karger AG, Basel

\section{Introduction}

Colorectal cancer (CRC) is a major cause of cancerrelated death worldwide, and the rate of mortality due to CRC has been increasing in Asia including Japan [1]. It is well accepted that the adenoma-carcinoma sequence is a

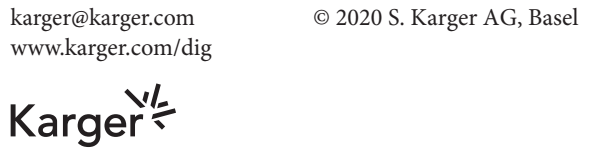

Tetsuji Takayama

Department of Gastroenterology and Oncology, Institute of Biomedical Sciences Tokushima University Graduate School

3-18-15, Kuramoto-cho, Tokushima 770-8503 (Japan)

takayama@tokushima-u.ac.jp 


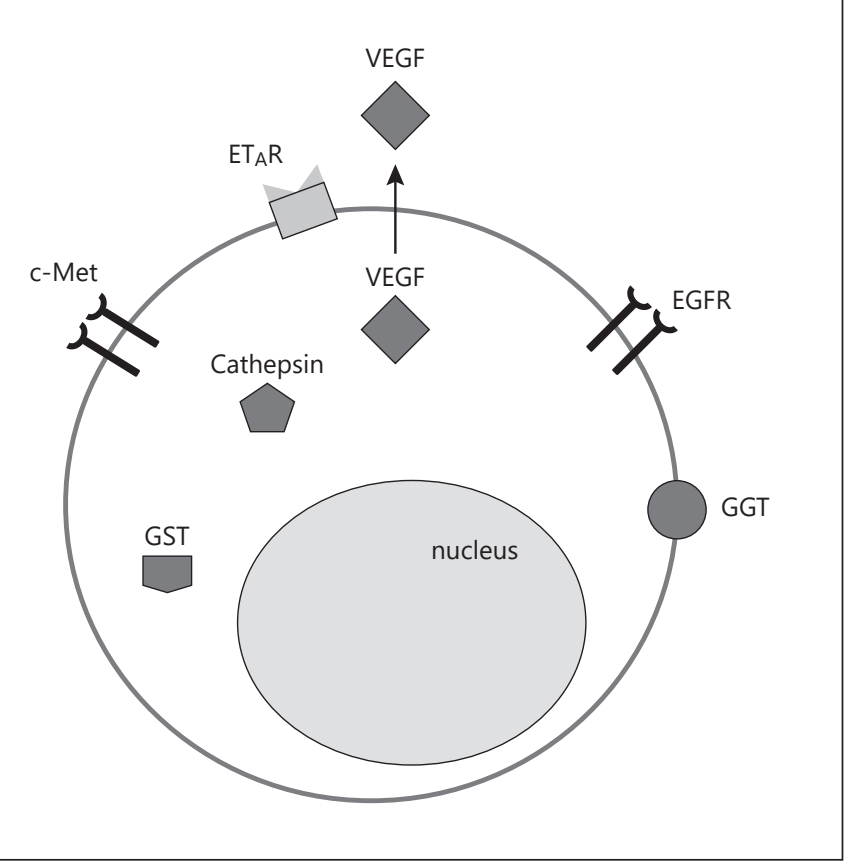

Fig. 1. Various targets for molecular imaging of colorectal tumors. VEGF, vascular endothelial growth factor; EGFR, epidermal growth factor receptor; $\mathrm{ET}_{\mathrm{A}} \mathrm{R}$, endothelin A receptor; GST, glutathione S-transferase; GGT, $\gamma$-glutamyltranspeptidase.

major pathway leading to CRC [2]. In addition, the serrated neoplasia sequence has been reported to be an alternative pathway to CRC. In patients with IBD, dysplasiacarcinoma sequence is recognized as an additional carcinogenesis pathway [3]. Therefore, it is very important to detect early-stage CRC and/or precancerous lesions such as adenoma, serrated polyps, and dysplasia in IBD patients through the use of colonoscopy. In general, white light (WL) colonoscopy is the gold standard for colonoscopy screening [4]. However, 15-32\% of adenomas go undetected with conventional colonoscopy, which is especially limited for detecting adenomas less than $10 \mathrm{~mm}$ in diameter or flat lesions [5]. With recent advances in the molecular biological analysis of colorectal tumors, molecular imaging, and fluorescence endoscopy targeting tumor-specific molecules are rapidly developing technologies that are expected to enable highly sensitive endoscopic detection of early neoplastic lesions. In this review, we summarize recent studies on the molecular imaging of colorectal tumors employing various types of fluorescence-labeled probes and discuss the current status of optical molecular imaging with fluorescence endoscopy for clinical application in the near future.

\section{Targets of Molecular Imaging in Colorectal Tumors}

The most important feature of a molecular imaging target is the highly specific expression in tumor cells. In the molecular imaging of colorectal tumors, the target molecule should ideally not only be overexpressed in tumors but also not expressed in surrounding nonneoplastic mucosa. Representative target molecules in colorectal tumors are shown in Figure 1. Epidermal growth factor receptor (EGFR), a receptor-type tyrosine kinase, is highly expressed in CRC and colorectal adenoma [6]. When ligands such as TGF- $\alpha$ or EGF bind to EGFR on the tumor cell surface, the EGFR molecule dimerizes and subsequent autophosphorylation of tyrosine kinase promotes signal transduction for tumor cell proliferation. Vascular endothelial growth factor (VEGF) is also highly expressed in CRC and colorectal adenoma tissue [7]. These tumor cells secrete VEGF to promote angiogenesis to receive nutrients and oxygen needed for rapid cell growth. Endothelin $A$ receptor $\left(\mathrm{ET}_{\mathrm{A}} \mathrm{R}\right)$ is one of the 2 receptor subtypes (A and $\mathrm{B}$ ) of the endothelin family [8]. $\mathrm{ET}_{\mathrm{A}} \mathrm{R}$ and its ligand are highly expressed in tumor cells including colorectal tumors, and their interaction induces tumor growth and metastasis by promoting tumor cell survival, proliferation, and angiogenesis.

In addition, enzymes such as glutathione S-transferase-P1 (GST-P1) in the cytosol, $\gamma$-glutamyl transpeptidase (GGT) in cell membranes, and cathepsin in lysosomes are highly expressed in certain tumor cell types including colorectal tumors $[9,10]$. Molecular imaging studies using a fluorogenic substrate probe, which is fluorescently quenched but activatable by enzyme activity only in the tumor cells, have been reported $[11,12]$.

\section{Fluorescent Probes for Colorectal Tumors}

Fluorescent probes are largely classified into 2 categories: employing an antibody against a tumor-specific molecule and small molecule compounds that specifically bind to target molecules in tumor cells.

\section{Fluorescence-Labeled Tumor-Specific Antibody}

Several antibodies, including anti-EGFR (cetuximab and panitumumab), anti-VEGF (bevacizumab), and antiVEGF receptor (ramucirumab) antibodies, have been approved for the therapy of metastatic CRC worldwide [13, 14]. They are currently recommended for the treatment of CRC as a standard regimen. Advantages of fluorescence-labeled antibody probes are that they are relatively 
Table 1. Endoscopic fluorescent molecular imaging for colorectal tumor in animals

\begin{tabular}{llllll}
\hline Lesion & Target & Species & Antibody/compound used & Fluorescence substance & Author \\
\hline CRC & EGFR & Mouse & Anti-EGFR-Ab (cetuximab) & AF488 & Goetz et al. [17] \\
CRC & VEGFA & Mouse & Anti-VEGF-Ab (bevacizumab) & ICG & Suo et al. [18] \\
Adenoma & EGFR & Rat & Anti-EGFR-Ab & AF488 & Miyamoto et al. [19] \\
Adenoma & c-Met & Mouse & Peptide ligand for c-Met & Cy5.5 & Wu et al. [20] \\
ACF & GST & Mouse & Substrate for GST & FITC & Muguruma et al. [11] \\
Dysplasia & GGT & Mouse & Substrate for GGT & ProteoGREEN & Mitsunaga et al. [16] \\
Dysplasia & Cathepsin & Mouse & Substrate for cathepsin & Cy5 & Gounaris et al. [12] \\
Dysplasia & ET $_{\mathrm{A}} \mathrm{R}$ & Mouse & ET $_{\mathrm{A}}$ R antagonist & Cy5.5 & Mücke et al. [22] \\
\hline
\end{tabular}

CRC, colorectal cancer; EGFR, epidermal growth factor receptor; VEGFA, vascular endothelial growth factor A; ICG, indocyanine green; ACF, aberrant crypt foci; GST, glutathione-S-transferase; GGT, $\gamma$-gultamyltranspeptidase; FITC, fluorescein isothiocyanate; $\mathrm{ET}_{\mathrm{A}} \mathrm{R}$, endothelin A receptor.

easy to apply in human clinical trials and that they show high specificity for the targeting molecule. However, disadvantages of them include a longer time (more than 24 h) to bind antigen sufficiently after intravenous administration, and immunogenicity.

\section{Fluorescence-Labeled Small Molecular Compounds}

Small molecular compounds, including enzyme substrates, synthetic peptides, and receptor antagonists, which bind to a specific target molecule in tumor cells, were labeled with fluorophore and used for molecular imaging. The fluorogenic substrate probe is a fluorescently quenched small molecule substrate designed to react with an enzyme in tumor cells to release a strong fluorophore. Fluorogenic probe reacting with GGT, GST, and cathepsin in tumor cells has been reported $[12,15$, 16]. Recently, a synthetic peptide has been used for molecular imaging studies. The peptide probe is usually developed using the phage display method against a specific protein expressed in tumor cells. Advantages of peptide probes include generally lower toxicity, lower immunogenicity, increased permeability, and flexibility in chemical modulation. Disadvantages of peptide probes include the labor intensiveness of identifying a specific peptide sequence during peptide synthesis and the need to sufficiently wash out residual fluorescent probe during colonoscopy.

\section{Molecular Imaging of Colorectal Tumors in Animals}

Representative endoscopic molecular imaging studies for detection of CRC and polyps in animals are summarized in Table 1. A CRC molecular imaging study using
anti-EGFR antibody (cetuximab) labeled with Alexa Fluor 488 (AF488) was reported by Goetz and associates [17]. They inoculated 2 human CRC cell lines (HT 29 cells, which strongly express EGFR, and SW600 cells, which weakly express EGFR) into nude mice, administered AF488-labeled cetuximab intravenously, and observed the tumors using confocal laser endoscopy (CLE) after $48 \mathrm{~h}$. Stronger fluorescence was observed in the xenograft tumors with HT29 cells than in SW600 cells. The HT29-xenografted mice showed significantly slower tumor growth and better survival than SW600-xenografted mice. Since cetuximab has strong anticancer activity against CRC cells, molecular imaging with fluorescencelabeled cetuximab is useful not only for tumor imaging but also for inhibiting tumor growth during the diagnostic examination.

Suo and colleagues labeled the anti-VEGFA antibody bevacizumab with indocyanine green (ICG) and administered the fluorescent probe intravenously in an orthotopic xenograft rat model of CRC [18]. A clear fluorescent image of the tumor was visualized using their self-made fluorescent endoscopy system for the near infrared spectral range.

Since adenoma-carcinoma is one of the major pathways of colorectal carcinogenesis, studies targeting adenomas have been reported. Miyamoto and associates performed molecular imaging of colorectal adenoma using AF488-labeled cetuximab in an azoxymethane (AOM)induced chemical carcinogenesis rat model [19]. They observed small adenomas on the colorectal mucosa of AOM-treated rats using veterinary endoscopy with WL. They, then, sprayed AF488-labeled cetuximab and observed the colorectal mucosa with a fluorescence endoscope. Strong fluorescent signals were seen on the mu- 
$(-)$

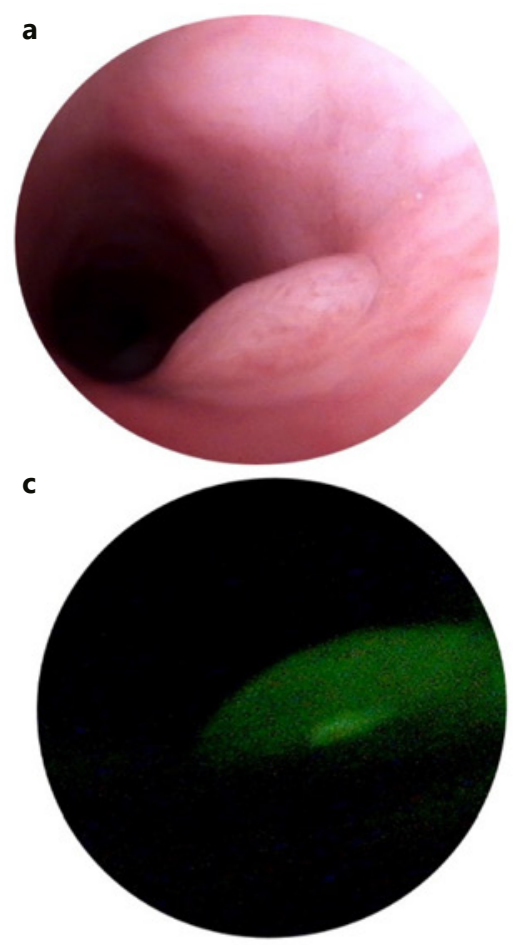

$(+)$

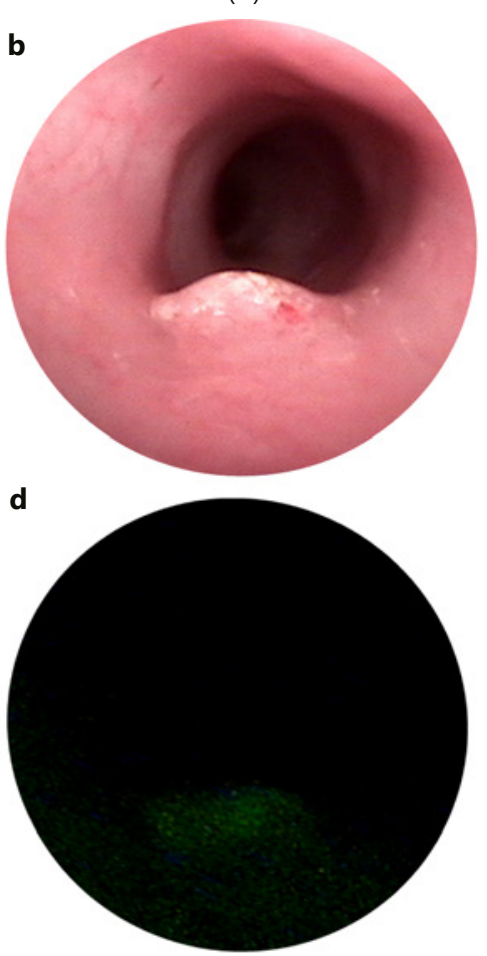

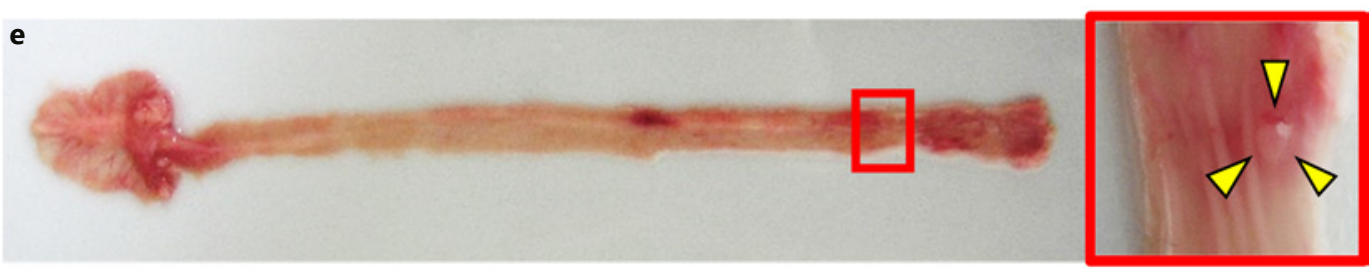

f

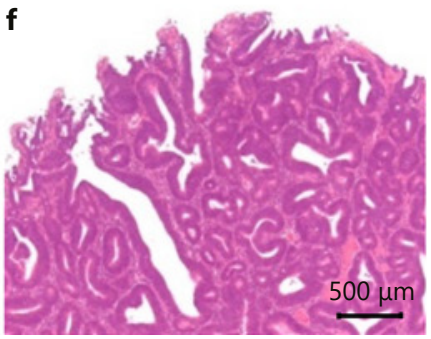

g

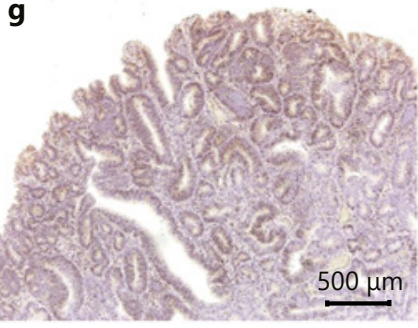

h

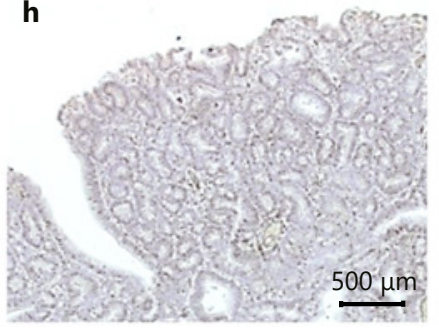

Fig. 2. Endoscopic and histological findings of colorectal polyps from AOM-treated rats. Representative endoscopic image of a colorectal polyp in an AOM-injected rat observed using a thin endoscope (a, b), A 3-mL aliquot of AF488-EGFR-Ab $(20 \mu \mathrm{g} / \mathrm{mL})$ was given by enema with or without pretreatment with non-labeled EGFR-Ab $(200 \mu \mathrm{g} / \mathrm{mL})(\mathbf{c}, \mathbf{d})$, and colorectal polyps were detected by fluorescent thin endoscopy (e). Histopathological findings of the resected tumor (h, e), EGFR immunohistochemical staining of the polyp (f). Figure 2 was adopted and modified from Fig. 5 in Cancer Sci. 2019;110:1921-30. AOM, azoxymethane; AF488, Alexa Fluor 488; EGFR, epidermal growth factor receptor. 
Table 2. Human clinical trial on endoscopic fluorescent molecular imaging for colorectal tumor

\begin{tabular}{|c|c|c|c|c|c|c|}
\hline Adenoma & c-Met & Peptide ligand for c-Met & Proprietary cyanine dye & 17 & Intravenous & Burggraaf et al. [23] \\
\hline Adenoma/SSL & VEGFA & Anti-VEGFA-Ab (bevacizumab) & RDye $800 \mathrm{CW}$ & 15 & Intravenous & Hartmans et al. [24] \\
\hline SSL & unknown & Peptide & FITC & 25 & Intrarectal & Joshi et al. [25] \\
\hline
\end{tabular}

SSL, sessile serrated lesion; FITC, fluorescein isothiocyanate; VEGFA, vascular endothelial growth factor A; ICG, indocyanine green. IRDye 800CW.

cosa at the same site (Fig. 2). All polyps found under WL, including small polyps with a dimeter of 1-2 $\mathrm{mm}$, were detectable by fluorescence imaging. Strong expression of EGFR was confirmed in the adenoma tissue.

$\mathrm{Wu}$ and associates developed a synthetic peptide ligand for c-Met using a phage library method and labeled it with Cy5.5 to synthesize a fluorescent peptide probe [20]. They administered it intrarectally in CPC-Apc mice, which spontaneously develop adenomatous polyps, and observed the colorectum. Fluorescence signals were observed in flat adenomas as well as in polypoid adenomas.

We found that GSTP1-1 is highly expressed in aberrant crypt foci (ACF) and colorectal adenoma as well as in CRC [9]. ACF is the earliest form of colorectal tumor lesion, which shows dense staining with methylene blue and is detectable by stereomicroscopy or magnifying endoscopy [21]. Employing a fluorogenic probe (DNAT-Me) activated by GSTP1-1, we performed molecular imaging of ACF, colorectal adenoma, and $\mathrm{CRC}$ in an AOM-induced rat carcinogenesis model and in human colorectum ex vivo [11]. DNAT-Me is a fluorescently quenched substrate but is activated by GSTP1-1 in tumor cells to release a strong fluorophore. We clearly visualized small ACF on the colorectal mucosa of AOM-treated rats. We also visualized human ACF on colorectal mucosa in surgically resected specimens from 7 CRC patients. After the operation, we sprayed DNAT-Me on the colorectal mucosa of resected tissue and successfully detected ACF using our fluorescence imaging system. Almost all ACF were detected with a strong signal, and the $\mathrm{S} / \mathrm{N}$ ratio of images taken with of DNAT-Me $(1.82 \pm 0.42)$ was significantly higher than that with methylene blue staining. We found that this technology may enable easy and sensitive detection of $\mathrm{ACF}$ as a surrogate marker for risk stratification and the evaluation of chemopreventive agents for CRC.

Dysplasia-carcinoma sequence is also a well-recognized pathway to CRC in IBD patients, and the molecular imaging studies for such colitis-associated cancers have been re-

Molecular Imaging of Colorectal Tumors ported. Mitsunaga and associates conducted an imaging studyusing thefluorogenicprobe $\gamma$-glutamylhydroxymethyl rhodamine green, which is cleaved by GGT to release the fluorescent compound rhodamine green $[15,16]$. The fluorogenic probe was administered by enema in mice treated with dextran sodium sulfate/AOM, and dysplastic lesions were observed using a veterinary fluorescence endoscopy. Several tumors on the colorectal mucosa were detectable by fluorescence endoscopy, and the histopathology of the dysplasia was confirmed by colonoscopic biopsy.

A fluorescently quenched substrate-based probe activated by cathepsin activity to release strongly fluorescent Cy5.5 was synthesized for molecular imaging of dysplastic lesions in ulcerative colitis based on previous findings showing that the lesions have enhanced cathepsin activity [12]. The fluorogenic probe was intravenously injected in IL-10-knockout mice, and fluorescent signals were visualized in the colorectal mucosa using a veterinary fluorescent endoscope. Histological examination revealed dysplastic lesions where cathepsin activity was increased.

Moreover, Mucke and associates conducted a study to visualize dysplasia and cancer targeting $\mathrm{ET}_{\mathrm{A}} \mathrm{R}$ in cell membranes [22]. Since $\mathrm{ET}_{\mathrm{A}} \mathrm{R}$ has been reported to be highly expressed in CAC tissues, they synthesized a fluorescent probe by labeling an $\mathrm{ET}_{\mathrm{A}} \mathrm{R}$ antagonist with Cy5.5 and administered it intravenously in a dextran sodium sulfate/AOM mouse model, followed by observation of the colorectal mucosa by fluorescence endoscopy. Tumors were easily detectable compared with the adjacent mucosa with significantly high contrast.

\section{Human Clinical Trial for Endoscopic Detection of Colorectal Tumors}

Three human clinical trials of endoscopic molecular imaging for colorectal tumors have been reported to date (Table 2). Burggraaf and associates developed a 26-amino 
acid cyclic peptide that specifically binds human c-MET employing the phage display method [23]. They labeled the peptide with cyanine dye (Cy5) (GE-137), injected it in mice in a xenograft model, and detected specific fluorescent signals on the tumor. After completing toxicity experiments in rats and monkeys, they performed a phase I study in humans to assess toxicity and determine optimal dosing. They administered the peptide probe intravenously in 15 patients at high risk of colorectal neoplasia and observed the colorectal mucosa using prototype endoscopy, which can generate fluorescence and WL images simultaneously. They were able to visualize all neoplastic polyps using fluorescent imaging, including 9 additional polyps that were not visible with WL.

Hartmans and associates performed a pilot study in humans using IRDye 800CW-labeled anti-VEGFA antibody (bevacizumab) [24]. They administered IRDye $800 \mathrm{CW}$-labeled bevacizumab intravenously in 17 patients with familial adenomatous polyposis (FAP), and observations were made 3 days later using both WL endoscopy and NIR-fluorescence endoscopy on the same monitor simultaneously. Even small adenomas less than $3 \mathrm{~mm}$ in diameter were detectable by fluorescent endoscopy with IRDye $800 \mathrm{CW}$ in colorectal mucosa.

Recently, Joshi and associates using a phage display method identified a peptide that binds specifically to sessile serrated lesions (SSLs) [25]. SSLs are precancerous lesions that predominantly develop in the proximal colon and are positive for BRAF V600E mutation. Although the target molecule of the peptide is unknown, they synthesized an FITC-labeled peptide probe and validated the specific binding of the labeled peptide with SSL cells in in vitro experiments. After conducting a toxicity study of the fluorescent probe in rats, they performed a pilot study in humans to test for safety and imaging of SSL. They observed the proximal colon using colonoscopy with WL, sprayed FITC-labeled peptide on the colorectal mucosa, and then performed fluorescence observation. They observed 19 SSLs in 38 patients with WL and fluorescent images. The mean fluorescence intensity in the SSLs was 2.43-fold higher than in surrounding nonneoplastic mucosa, and SSLs were distinguishable from nonneoplastic mucosa with $89 \%$ sensitivity and $92 \%$ specificity.

\section{Fluorescent Dyes}

Near infrared light has a wavelength between $700 \mathrm{~nm}$ and $1 \mu \mathrm{m}$. It is useful in terms of its higher tissue penetration and safety for human body than ultraviolet rays.
Moreover, there is little background noise in the human body, especially in the gastrointestinal tract under infrared light exposure [26]. The interest in the use of nearinfrared fluorescence imaging has been steadily growing.

ICG has been used in humans for more than 50 years. ICG emits light at $807-832 \mathrm{~nm}$ upon excitation at around $770 \mathrm{~nm}$ [27]. Although the structure of ICG must be modified to enable covalent binding with an antibody or small molecule, it might be suitable as a molecular imaging agent due to its low toxicity. IRDye 800CW (IR800) is a water-soluble near-infrared dye with a peak excitation at $774 \mathrm{~nm}$ and emission at $803 \mathrm{~nm}$ [28]. IRDye $800 \mathrm{CW}$ has ideal quantum yields and a high extinction coefficient, in addition to being more photostable and brighter.

Fluorescein and its derivative FITC have been used in humans for more than 30 years. FITC has an excitation peak at $498 \mathrm{~nm}$ and an emission peak at $522 \mathrm{~nm}$. Although FITC is inexpensive and widely used for molecular imaging, its disadvantages include lower tissue penetration due to the relative short wavelength $(517 \mathrm{~nm})$ and a short decay time.

Other fluorescent dyes, including Alexa Fluor and Cy5 are used for molecular imaging studies. For the clinical use of these dyes, characteristics including toxicity, stability, and fluorescent intensity in the human body need to be analyzed.

\section{Future Areas of Investigation}

We did not describe herein endoscopic devices for fluorescent observation in detail. There are currently no commercially available fluorescent endoscopy device with sufficient performance. Therefore, in the human studies of endoscopic molecular imaging described above, the authors and collaborative groups used self-made endoscopic devices or prototype endoscopic systems. CLE is an emerging technology which allows real-time in vivo imaging of cellular and subcellular details of the mucosa and vessels with high-resolution images. CLE could be applicable to endoscopic molecular imaging [17] because of the optical characteristics, including deeper observation depth range $(0-250 \mu \mathrm{m})$. However, this device is not currently suitable for screening endoscopy because of the limited field of view $(475 \times 475 \mu \mathrm{m})$. Moreover, the laser scanning unit is limited to the excitation wavelength of $488 \mathrm{~nm} / 660 \mathrm{~nm}$, and more flexibility for various fluorescent agents such as IRDye and ICG is required. Thus, it is important to develop endoscopic devices with sufficiently high performance and the ability to observe various wavelengths.
Kashihara/Muguruma/Fujimoto/ Miyamoto/Sato/Takayama 


\section{Requirement for Clinical Application and Limitation of Molecular Imaging}

To apply the molecular imaging technology to clinical endoscopic examination, a large-scale clinical trial to test the utility and safety of the fluorescent probe is needed. The detection rate with molecular imaging using the fluorescent probe should be compared to those with WL and image-enhanced endoscopy such as narrow banding image and Blue laser image. Moreover, as described above, a high-performance fluorescent endoscopic device should be developed.

There are currently some limitations of molecular imaging technology. First, it needs administration of fluorescent probe into human body via either intravenous administration or topical administration. Therefore, we have to confirm the safety of the fluorescent probe. Second, the molecular target is different among the precancerous lesions such as adenoma, serrated polyps, and dysplasia, and therefore, currently there is no fluorescent probe which is able to detect all the precancerous lesion types simultaneously.

\section{Conclusion}

Molecular imaging endoscopy is expected to play an important role in the diagnosis of colorectal tumors and in guiding decision-making regarding the choice of treatment, as well as to provide a means for early-phase treatment of colorectal tumors. The development of this technology is in its early stages, and several challenges remain before it can be brought to fruition, including target identification, compound screening, preclinical testing, and phase 1-3 trials. If successful, this technology could lead to great progress in clinical oncology.

\section{Conflict of Interest Statement}

The authors have no conflicts of interest to declare.

\section{Funding Sources}

The authors have no finding sources.

\section{Author Contributions}

T. Takayama designed the structure of the manuscript. T. Kashihara mainly wrote the draft manuscript. N. Muguruma described the fluorescent probe section. S. Fujimoto described the animal experiment section. Y. Miyamoto described the fluorescent dye section. Y. Sato was responsible for figures and tables and references.

\section{References}

1 Torre LA, Siegel RL, Ward EM, Jemal A. Global cancer incidence and mortality rates and trends: an update. Cancer Epidemiol Biomarkers Prev. 2016;25(1):16-27.

2 Leslie A, Carey FA, Pratt NR, Steele RJ, Leslie MA. The colorectal adenoma-carcinoma sequence. Br J Surg. 2002;89(7):845-60.

3 Itzkowitz SH, Yio X. Inflammation and cancer IV. Colorectal cancer in inflammatory bowel disease: the role of inflammation. Am J Physiol Gastrointest Liver Physiol. 2004; 287(1):G7-17.

4 Lieberman DA, Weiss DG, Bond JH, Ahnen DJ, Garewal H, Chejfec G. Use of colonoscopy to screen asymptomatic adults for colorectal cancer. Veterans affairs cooperative study group 380. N Engl J Med. 2000;343(3):162-8.

5 Van Rijn JC, Reitsma JB, Stoker J, Bossuyt PM, Van Deventer SJ, Dekker E. Polyp miss rate determined by tandem colonoscopy: a systematic review. Am J Gastroenterol. 2006; 101(2):343-50.

6 Normanno N, De Luca A, Bianco C, Strizzi L, Mancino M, Maiello MR, et al. Epidermal growth factor receptor (EGFR) signaling in cancer. Gene. 2006;366(1):2-16.

7 Brown LF, Berse B, Jackman RW, Tognazzi K, Guidi AJ, Dvorak HF, et al. Expression of vascular permeability factor (vascular endothelial growth factor) and its receptors in breast cancer. Hum Pathol. 1995;26(1):86-91.

8 Grant K, Loizidou M, Taylor I. Endothelin-1: a multifunctional molecule in cancer. $\mathrm{Br} \mathrm{J}$ Cancer. 2003;88(2):163-6.

9 Miyanishi K, Takayama T, Ohi M, Hayashi T, Nobuoka A, Nakajima T, et al. Glutathione Stransferase-pi overexpression is closely associated with K-ras mutation during human colon carcinogenesis. Gastroenterology. 2001; 121(4):865-74.

10 Pompella A, De Tata V, Paolicchi A, Zunino F. Expression of gamma-glutamyltransferase in cancer cells and its significance in drug resistance. Biochem Pharmacol. 2006;71(3): 231-8.

11 Muguruma N, Okamoto K, Nakagawa T, Sannomiya K, Fujimoto S, Mitsui Y, et al. Molecular imaging of aberrant crypt foci in the human colon targeting glutathione S-transferase P1-1. Sci Rep. 2017;7(1):6536.

12 Gounaris E, Martin J, Ishihara Y, Khan MW, Lee G, Sinh P, et al. Fluorescence endoscopy of cathepsin activity discriminates dysplasia from colitis. Inflamm Bowel Dis. 2013;19(7): 1339-45.

13 Van Cutsem E, Köhne CH, Hitre E, Zaluski J, Chang Chien CR, Makhson A, et al. Cetuximab and chemotherapy as initial treatment for metastatic colorectal cancer. N Engl J Med. 2009;360(14):1408-17.

14 Kabbinavar F, Hurwitz HI, Fehrenbacher L, Meropol NJ, Novotny WF, Lieberman G, et al. Phase II, randomized trial comparing bevacizumab plus fluorouracil (FU)/Leucovorin (LV) with $\mathrm{FU} / \mathrm{LV}$ alone in patients with metastatic colorectal cancer. J Clin Oncol. 2003;21:60-5.

15 Urano Y, Sakabe M, Kosaka N, Ogawa M, Mitsunaga M, Asanuma D, et al. Rapid cancer detection by topically spraying a $\gamma$-glutamyltranspeptidase-activated fluorescent probe. Sci Transl Med. 2011;3(110): 110ra119-9. 
16 Mitsunaga M, Kosaka N, Choyke PL, Young MR, Dextras CR, Saud SM, et al. Fluorescence endoscopic detection of murine colitis-associated colon cancer by topically applied enzymatically rapid-activatable probe. Gut. 2013; 62(8):1179-86.

17 Goetz M, Hoetker MS, Diken M, Galle PR, Kiesslich R. In vivo molecular imaging with cetuximab, an anti-EGFR antibody, for prediction of response in xenograft models of human colorectal cancer. Endoscopy. 2013; 45(6):469-77.

18 Suo Y, Wu F, Xu P, Shi H, Wang T, Liu H, et al. NIR-II fluorescence endoscopy for targeted imaging of colorectal cancer. Adv Healthc Mater. 2019;8(23):e1900974.

19 Miyamoto Y, Muguruma N, Fujimoto S, Okada Y, Kida Y, Nakamura F, et al. Epidermal growth factor receptor-targeted molecular imaging of colorectal tumors: detection and treatment evaluation of tumors in animal models. Cancer Sci. 2019;110(6):1921-30.
20 Wu X, Zhou J, Wang F, Meng X, Chen J, Chang TS, et al. Detection of colonic neoplasia in vivo using near-infrared-labeled peptide targeting cMet. Sci Rep. 2019;9(1):17917.

21 Takayama T, Katsuki S, Takahashi Y, Ohi M, Nojiri S, Sakamaki S, et al. Aberrant crypt foci of the colon as precursors of adenoma and cancer. N Engl J Med. 1998;339(18):1277-84.

22 Mücke MM, Bettenworth D, Geyer C, Schwegmann K, Poremba C, Schäfers M, et al. Targeting mucosal endothelin-A-receptor expression by fluorescence endoscopy is feasible to detect and characterize colitis-associated cancer in mice. Inflamm Bowel Dis. 2018; 24(1):111-22.

23 Burggraaf J, Kamerling IM, Gordon PB, Schrier L, De Kam ML, Kales AJ, et al. Detection of colorectal polyps in humans using an intravenously administered fluorescent peptide targeted against c-Met. Nat Med. 2015; 21(8):955-61.
24 Hartmans E, Tjalma JJJ, Linssen MD, Allende PBG, Koller M, Jorritsma-Smit A, et al. Potential red-flag identification of colorectal adenomas with wide-field fluorescence molecular endoscopy. Theranostics. 2018;8(6):145867.

25 Joshi BP, Dai Z, Gao Z, Lee JH, Ghimire N, Chen J, et al. Detection of sessile serrated adenomas in the proximal colon using widefield fluorescence endoscopy. Gastroenterology. 2017;152(5):1002-e9.

26 Muguruma N, Miyamoto H, Okahisa T, Takayama T. Endoscopic molecular imaging: status and future perspective. Clin Endosc. 2013;46(6):603-10.

27 Mordon S, Devoisselle JM, Soulie-Begu S, Desmettre T. Indocyanine green: physicochemical factors affecting its fluorescence in vivo. Microvasc Res. 1998;55(2):146-52.

28 Marshall MV, Draney D, Sevick-Muraca EM, Olive DM. Single-dose intravenous toxicity study of IRDye $800 \mathrm{CW}$ in sprague-dawley rats. Mol Imaging Biol. 2010;12(6):583-94. 